\title{
Contrast Agent Choice for Intravenous Coronary Angiography
}

\author{
H.D. ZEMAN AND D.P. SIDDONS \\ National Synchrotron Light Source \\ Brookhaven National Laboratory \\ Upton, New York 11973
}

\begin{abstract}
The screening of the general population for coronary artery disease would be practical if a method existed for visualizing the extent of occlusion after an intravenous injection of contrast agent. Measurements performed with monochromatic synchrotron radiation $\mathrm{X}$-rays and an iodine containing contrast agent at the Stanford Synchrotron Radiation Laboratory have shown that such an intravenous angiography procedure would be possible with an adequately intense monochromatic $\mathrm{X}$-ray source. Because of the size and cost of synchrotron radiation facilities it would be desirable to make the most efficient use of the intensity available, while reducing as much as possible the radiation dose experienced by the patient. By choosing contrast agents containing elements with a higher atomic number than iodine, it is possible to both improve the image quality and reduce the patient radiation dose, while using the same synchrotron radiation source. By using $\mathrm{Si}$ monochromator crystals with a small mosaic spread, it is possible to increase the $\mathrm{X}$-ray flux available for imaging by over an order of magnitude, without any changes in the storage ring or wiggler magnet.
\end{abstract}

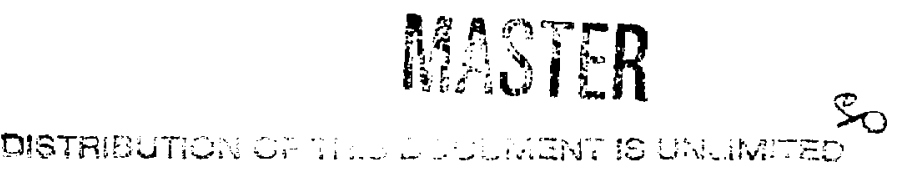


The most critical imaging task for intravenous coronary angiography utilizing synchrotron radiation $\mathrm{X}$-rays is visualizing a coronary artery through the left ventricle or aorta which also contains contrast agent. Calculations have been made of the signal to noise ratio expected for this imaging task for various contrast agents with atomic numbers between that of iodine and bismuth. The X-ray energy spectrum of the $\mathrm{X}-17$ superconduction wiggler beam line at the National Synchrotron Light Source at Brookhaven National Laboratory has been used for these calculations. Both perfect Si crystals and Si crystals with a small mosaic spread are considered as monochromators. Contrast agents containing $\mathrm{Gd}$ or $\mathrm{Yb}$ seem to have about the optimal calculated signal to noise ratio. Gd-DTPA is already approved for use as a contrast agent for magnetic resonance imaging (MRI). Experiments have already been performed with Yb-DTPA in animals, and it appears to have a lower toxicity than that of Gd-DTPA. Reported animal experiments with GdDOTA contrast agent show no toxicity at all. 


\section{Introduction}

The concept behind using monochromatic $X$-ray beams to image injected contrast agent intravenously has been described previously. The human angiographic images taken so far at the Stanford Synchrotron Radiation Laboratory (SSRL) with iodine contrast agent ${ }^{2}$ show great promise, and the higher X-ray intensity that will be available at the superconducting wiggler beam line ${ }^{3}$ at the National Synchrotron Light Source (NSLS) at Brookhaven National Laboratory should allow images to be produced with intravenous injection of iodine contrast agent that approach the quality of images produced with selective arterial injection. However, the image quality and patient radiation dose at NSLS will depend strongly on the choice of contrast agent element, and the type of X-ray monochromator crystals used. In the following, the criteria for choosing an optimal contrast agent element will be developed. The properties of the superconducting wiggler beam line (X-17) at NSLS will be used for this analysis, along with the reflecting properties of both perfect Si crystals and Si crystals with a small mosaic spread.

\section{Theory}

\subsection{Signal to Noise Ratio for Viewing an Artery Throvgh a Filled VENTRICLE}

In the synchrotron radiation technique described in Ref. 1 two separate monochromatic X-ray beams are used, one with an energy above and one with an energy below the K-edge of the contrast agent. Measurements are taken through the patient with each beam separately. When a large amount of contrast agelt is present in the left ventricle, the above $K$-edge beam is much more strongly absorbed than 
the below K-edge beam. To calculate the signal to noise ratio of a measurement of the thickness of an artery which lies behind the filled left ventricle, the X-ray beams will be assumed to lie exactly above and below the K-edge, and the beams will be assumed to be exactly monochromatic.

Let $N_{1}$ be the number of X-ray photons per pixel incident on the patient with an energy above the K-edge of the contrast agent. Let $N_{2}$ be the same below the K-edge. Let $\mu_{1}$ be the mass attenuation coefficient of the contrast agent above its K-edge, and $\mu_{2}$ the same below. Let $T_{V}$ be the thickness of the contrast agent in the ventricle in $\mathrm{g} / \mathrm{cm}^{2}$, and $T_{A}$ be the same for the artery behind it. Let

$$
T_{A}=f T_{V}
$$

where $f$ is a small constant. Let $\mu_{B}$ be the average mass attenuation coefficient of the patient, and let $T_{B}$ be the thickness of the patient in $\mathrm{g} / \mathrm{cm}^{2}$.

Four measurements of the number of photons transmitted through the patient per pixel are required to determine the thickness of the artery. One measurement below the K-edge is taken through the patient the ventricle and the artery $\left(N_{22}\right)$. Another measurement below the K-edge is taken through the patient and the ventricle, but just next to and missing the artery $\left(N_{21}\right)$. Similarly above the Kedge two more measurements are taken, one through the artery $\left(N_{12}\right)$, and one next to it $\left(N_{11}\right)$. These four measurements would yield the following:

$$
\begin{gathered}
N_{11}=N_{1} e^{-\mu_{B} T_{B}} e^{-\mu_{1} T_{v}}, \\
N_{12}=N_{11} e^{\mu_{1} f T_{v}},
\end{gathered}
$$




$$
N_{21}=N_{2} e^{-\mu_{B} T_{B}} e^{-\mu_{2} T_{V}}
$$

and

$$
N_{22}=N_{21} e^{\mu_{2} f T V} .
$$

To eliminate the contrast due to the thickness of the body, the following ratios are formed:

$$
\frac{N_{11}}{N_{21}}=\frac{N_{1}}{N_{2}} e^{\left(\mu_{1}-\mu_{2}\right) T},
$$

and

$$
\frac{N_{12}}{N_{22}}=\frac{N_{1}}{N_{2}} e^{\left(\mu_{1}-\mu_{2}\right)(1+f) T_{\nu}}
$$

Expanding to first order for small $f$ we have

$$
\left(\mu_{1}-\mu_{2}\right) f T_{V}=1-\left(\frac{N_{12} N_{21}}{N_{22} N_{11}}\right)
$$

The statistical error in each of the measured numbers is simply the square root of the number. If the values of these square roots are taken from Eqns. (2.2)-(2.5), and use is made of the fact that $f$ is small, then an expression can be derived for the signal to statistical noise ratio for the measurement of $f T_{V}$, namely:

$$
S N R=K \frac{\sqrt{U(1-U)} A e^{-A}}{\sqrt{(1-U)+U e^{-2(1-N) A}}}
$$

where

$$
\begin{gathered}
K=\sqrt{2 N_{T}} f(1-N) e^{-\frac{1}{2} \mu_{B} T_{B}}, \\
N_{T}=N_{1}+N_{2},
\end{gathered}
$$




$$
\begin{gathered}
U=\frac{N_{1}}{N_{T}}, \\
A=\frac{1}{2} \mu_{1} T_{V},
\end{gathered}
$$

and

$$
N=\frac{\mu_{2}}{\mu_{1}}
$$

$N_{T}$ is the total number of X-ray photons incivent on the patient per pixel, and is therefore proportional to the patient X-ray dose. It would be desirable to know for what values of $U$ and $A$ a maximum value of $S N R$ would be found. If we set the partial derivative of $S N R$ with respect to $U$ equal to zero and solve for $U$ we have

$$
U=\frac{1}{1+e^{-(1-N) A}}
$$

Combining Eqns. (2.9)\&(2.15) we get

$$
S N R=K \frac{A e^{-A}}{1+e^{-(1-N) A}}
$$

If we set the partial derivative of $S N R$ as given in Eqn. (2.16) with respect to A equal to zero, we get the following transcendental equation:

$$
\frac{A-1}{1-N A}=e^{-(1-N) A}
$$

Eqn. (2.17) can be solved numerically to yield a value of $A$, which can be used in Eqn. (2.15) to give a value of $U$. The values of $A$ and $U$ can be used in Eqn. (2.9) to yield the maximum value of $S N R$. 
The results of the calculations described above are shown in Table I for the elements between $\mathrm{I}$ and $\mathrm{Bi}$. In evaluating $K$, values had to be chosen for the various constants. The value $N_{T}=10^{6}$ was chosen, since this value could easily be obtained at NSLS for any contrast agent from I to $\mathrm{Bi}$, assuming a $4 \mathrm{msec}$ exposure time and a $0.25 \times 0.25 \mathrm{~mm}$ pixel. For $\mu_{B}$ the mass attenuation coefficient of water at K-edge energy of the contrast agent was chosen. $N$ was also taken for the various contrast agent elements from Ref. 4 . The mass attenuation coefficients vs. X-ray energy for four representative contrast agents ( $\mathrm{I}, \mathrm{Gd}, \mathrm{Hf}$, and $\mathrm{Bi}$ ) along with bone and water are shown in Fig. 1. For $T_{B}$ the value of $20 \mathrm{~cm}$ of water was chosen, which is a realistic value for an average weight patient. The thinnest patient studied at SSRL had a thickness equivalent to $15.6 \mathrm{~cm}$ of water. Heavier patients must be able to be studied if the technique is ever to have wide applicability. The value of $f$ was chosen to be 0.1 (i.e. 10\%). This is a reasonable estimate of the thickness of a coronary artery large enough to be of diagnostic interest, placed in front of the left ventricle which had already begun to empty. The $S N R$ values shown in Table I are the optimal signal to noise ratios calculated using Eqns. (2.9),(2.15),\&(2.17).

The results from Table I are shown graphically in Fig. 2. It can be seen that the SNR clearly increases with K-edge energy, and is greatest with a bismuth contrast agent.

To show how the SNR varies with contrast agent concentration, the results from Eqn. (2.16) are shown in Fig. 3. The great improvement in SNR seen for the three heavier contrast agents as compared with iodine is entirely due to the reduced attenuation of the primary X-ray beam by the body at higher energy. This reduced attenuation also has the desirable result that image contrast due to the contrast agent will be a larger faction of the contrast due to soft tissue and bone, 
which will allow a detector with a smaller dynamic range to be used.

\subsection{X-Ray Flux Enhancement with Si Mosaic Crystal Monochro-} MATORS

For the synchrotron radiation angiographic imaging experiments performed at $\mathrm{SSRL}^{5}$ so far and planned for $\mathrm{NSLS}^{6}$ starting this fall, two separate single monochromator crystals are used to produce the two narrow energy band X-ray beams, one with an energy below the K-edge of the contrast agent and one with an energy above. If mosaic crystals are used in place of the presently used perfect crystals, a greater X-ray flux will be available for iniaging and a higher signal to noise ratio will be possible. To calculate the ratio of the flux available from a $\mathrm{Si}$ mosaic crystal as compared to a perfect $\mathrm{Si}$ crystal, the theoretical analysis of Freund $^{7}$ for mosaic crystals is compared with the analysis of perfect crystals by Beaumont and Hart.

The reflectivity for a mosaic crystal with mosaic distribution half-width $\Gamma$ is given by Freund as a function of the deviation angle $\delta$ as

$$
r=\frac{a}{1+a+\sqrt{1+2 a} \cdot \operatorname{coth}(A \cdot \sqrt{1+2 a})},
$$

where

$$
\begin{gathered}
a=\frac{\omega \cdot Q}{\mu}, \\
A=\frac{\mu \cdot t}{\sin \theta}, \\
\omega=\frac{\exp C}{\eta \cdot \sqrt{2 \cdot \pi}},
\end{gathered}
$$




$$
\begin{gathered}
C=\frac{-\delta^{2}}{2 \eta^{2}}, \\
Q=\left(\frac{F_{x}}{\alpha^{3}}\right)^{2} \cdot \frac{\lambda^{3}}{\sin \theta}, \\
\theta=\arcsin \left(\frac{\lambda}{2 \cdot d}\right), \\
F_{x}=\left(\frac{e^{2}}{m c^{2}}\right) \cdot F_{h} \cdot \exp \left(\frac{-B}{4 d^{2}}\right), \\
\eta=\frac{\Gamma}{2.35}
\end{gathered}
$$

and

$$
d=\frac{\alpha}{\sqrt{3}}
$$

The numerical constants for Si are $\alpha=5.431065 \AA, B=0.461 \AA^{2}, V=160 \AA^{3}$, $F_{h}=60.13, \epsilon_{0}=0.963$, and $Z=14$.

The reflectivity of a perfect crystal is given by Beaumont and Hart as a function of the deviation angle $\delta$ as

$$
R=E-\sqrt{E^{2}-1}
$$

where

$$
\begin{gathered}
E=D^{2}+F^{2}+\sqrt{1+\left(D^{2}+F^{2}\right)^{2}-2 \cdot\left(D^{2}-F^{2}\right)}, \\
D=\frac{\left|\chi_{i 0}\right|}{\left|\chi_{r h}\right|} \cdot \frac{\chi_{i h}}{\chi_{r h}}+y, \\
F=\frac{\left|\chi_{i 0}\right|}{\left|\chi_{r h}\right|}-\frac{\chi_{i h}}{\chi_{r h}} \cdot y,
\end{gathered}
$$




$$
\begin{gathered}
\chi_{r 0}=\left(\frac{-e^{2}}{\pi m c^{2}}\right) \cdot \lambda^{2} \cdot \frac{8 Z}{V}, \\
\chi_{r h}=\left(\frac{-e^{2}}{\pi m c^{2}}\right) \cdot \lambda^{2} \cdot \frac{F_{h}}{V}, \\
\chi_{i 0}=\frac{-\lambda \mu}{2 \pi} \\
\chi_{i h}=\chi_{i 0} \epsilon_{0} \exp \left(\frac{-B}{4 d^{2}}\right),
\end{gathered}
$$

and

$$
y=\frac{\delta \sin 2 \theta_{0}}{\left|\chi_{r h}\right|}
$$

The quantities $r$ and $R$ from Eqns. (2.18)\&(2.28) were integrated over all deviation angles $\delta$ for various mosaic distribution half-widths $\Gamma$. The ratio of these integrals gives a good estimate of the enhancement of the available X-ray flux by using mosaic crystals. The results of this calculation for several values of $\Gamma$ are shown in Fig. 4. Note how the enhancement improves with increasing $\Gamma$ in this range ( 1 to 15 arc seconds).

\section{Criteria for Contrast Agent Choice}

\subsection{Contrast Agent Concentration}

If it were possible to achieve any contrast agent concentration one wanted in the left ventricle and the coronary arteries after an intravenous injection, then clearly Fig. 2 would indicate that $\mathrm{Bi}$ would be the best contrast agent choice. However Fig. 1 shows that the mass attenuation coefficient for the various contrast agents just above the $\mathrm{K}$-edge decreases rapidly with increasing $\mathrm{K}$-edge energy. Hence to 
achieve the optimal concentration for a heavier contrast agent, a significantly higher mass of the agent would be needed. Eventually the effects of increased toxicity and reduced solubility will make such a high contrast agent concentration impossible. The square marks on Fig. 3 show the locations on the SNR ys. concentration curves where the same mass of contrast agent is injected as would be optimal for iodine. From measurements at SSRL it is clear that a concentration of iodine contrast agent can be achieved in the left ventricle that is greater than the optimal value given in Table I. If toxicity and solubility are generally a function of $\mathrm{mg} / \mathrm{kg}$ of body weight and $\mathrm{mg} / \mathrm{ml}$ of water, respectively, then it is not unreasonable to consider the SNR achievable for a constant number of mg of contrast agent. A curve of SNR vs. the atomic number of the contrast agent is given in Fig. 5 where a constant mass of contrast agent is assumed. The lower curve is also for a constant photon count per pixel. For the upper curve the total X-ray dose absorbed in the $20 \mathrm{~cm}$ of water is constant, with $10^{6}$ photons used for iodine, and more for the other elements. Note that at constant contrast agent mass and constant radiation dose, the heaviest contrast agents are best just like in Fig. 2.

\subsection{Signal to NoISE for the X-17 Superconducting Wiggler Source} AT NSLS

If cost were no object in designing an X-ray source for angiography, then the considerations above would suggest that bismuth would give the best image quality for the same patient dose. However, the properties of real sources of monochromatic $\mathrm{X}$-rays have to be considered, if a practical compromise between cost and patient dose is to be found. Also a more accurate analysis of the toxicity and solubility problems would really be needed. 
In Fig. 6 the output of the X-17 superconducting wiggler magnet synchrotron radiation source at NSLS is shown. A 4 msec exposure time per $0.25 \times 0.25 \mathrm{~mm}$ pixel is assumed. Two curves are show, one for a perfect crystal monochromator and the other for a mosaic crystal. The mosaic distribution half-angle was chosen as 3 arc seconds to correspond with the largest value so far achieved with the method reported in the literature. ${ }^{9,10}$ As $\mathrm{Si}$ crystals with larger mosaic spreads become available, Fig. 4 shows that still higher $\mathrm{X}$-ray intensity would be possible. The signal to noise ratios achievable with the X-17 wiggler source for the various contrast agents are shown in Fig. 7. Note that the use of a gadolinium $(Z=64)$ or ytterbium ( $Z=70$ ) contrast agent would be near to optimum.

\subsection{Patient Skin Dose}

Besides the increased signal to noise ratio possible with higher $\mathrm{Z}$ contrast agents as compared with the iodine containing contrast agents presently used for angiography, a significantly lower patient skin dose is an additional benefit. The variation of skin dose with X-ray energy is shown in Fig. 8. The dose was calculated using the absorption coefficient of air ${ }^{11}$ to calculate the exposure in Roentgens, and the conversion factor for muscle to convert Roentgens of exposure to Rads of dose. As can be seen from Fig. 8 the skin dose per frame is significantly lower for a gadolinium ( $50 \mathrm{keV})$ or ytterbium (61 keV) contrast agent than it would be for iodine $(33 \mathrm{keV})$ while the signal to noise ratio shown in Fig. 7 is significantiy nigher for these higher $\mathrm{Z}$ contrast agents. 


\subsection{Contrast Agent Toxicity}

Gadolinium-DTPA is already approved by the FDA as a contrast agent for magnetic resonance imaging (MRI). Hence, it would be particularly desirable to be able to use gadolinium as a contrast agent for angiography as well. It is also of great interest that experiments have already been performed with a ytterbiumDTPA as a potential intravascular contrast agent. ${ }^{12}$ It appears that ytterbiumDTPA is somewhat less toxic than gadolinium-DTPA. However, ytterbium-DTPA is about four times more toxic per gram of contrast agent element than iodine containing Renografin. If one assumes that these chelated heavy element compounds are somewhat toxic because the chelating agent is not stable enough to prevent the heavy element from being released into the body before the chelated compound is eliminated by the kidneys, then it would be reasonable to imagine that a more stable compound would be less toxic. In a second reference, ${ }^{13}$ comparative measurements of stability are described where a new compound gadolinium-DOTA is shown to be $10^{6}$ times as stable as the gadolinium-DTPA presently approved for use in MRI. The authors also report seeing no toxic affects whatever on the animals used in the study. If the toxicity decreases $2 t$ all as the stability increases, then it is possible that Gd-DOTA and/or Yb-DOTA might make far less toxic contrast agents for angiography than Renografin. 


\section{Acknowledgments}

The support and encouragement of E. Rubenstein, W. Themlinson, and J. Hastings during this work is greatly appreciated. This work was performed under the auspices of the US Department of Energy under Grant No. DE-FG03-87ER60527 to the Stanford University Medical Center and under Contract No. DEAC02-76CH-00016 to the National Synchrotron Light Source, Brookhaven National Laboratory. 
Table I

The optimal values of the dimensionless quantities $U$ and $A$

defined in Eqns. (2.12) \& (2.19) and the resulting value of $S N R$

for various contrast agents. The values of the dimensionless

quantity $N$ defined in Eqn. (2.14) are from Ref. 4.

\begin{tabular}{|c|c|c|c|c|c|}
\hline Contrast & $\mathrm{Z}$ & $N$ & $U$ & $A$ & $S N R$ \\
\hline $\mathrm{I}$ & 53 & 0.182 & 0.7389 & 1.2716 & 0.109 \\
\hline $\mathrm{Xe}$ & 54 & 0.183 & 0.7386 & 1.2715 & 0.132 \\
\hline $\mathrm{Cs}$ & 55 & 0.184 & 0.7383 & 1.2714 & 0.153 \\
\hline $\mathrm{Sm}$ & 62 & 0.195 & 0.7356 & 1.2705 & 0.282 \\
\hline Eu & 63 & 0.196 & 0.7352 & 1.2704 & 0.297 \\
\hline $\mathrm{Gd}$ & 64 & 0.198 & 0.7347 & 1.2702 & 0.311 \\
\hline $\mathrm{Tb}$ & 65 & 0.200 & 0.7342 & 1.2701 & 0.325 \\
\hline $\mathrm{Dy}$ & 66 & 0.202 & 0.7337 & 1.2699 & 0.338 \\
\hline $\mathrm{Ho}$ & 67 & 0.204 & 0.7332 & 1.2697 & 0.350 \\
\hline $\mathrm{Er}$ & 68 & 0.206 & 0.7327 & 1.2695 & 0.361 \\
\hline $\mathrm{Lu}$ & 71 & 0.212 & 0.7309 & 1.2689 & 0.391 \\
\hline $\mathrm{Hf}$ & 72 & 0.215 & 0.7302 & 1.2686 & 0.400 \\
\hline $\mathrm{Ta}$ & 73 & 0.218 & 0.7296 & 1.2684 & 0.409 \\
\hline $\mathrm{W}$ & 74 & 0.220 & 0.7289 & 1.2681 & 0.416 \\
\hline $\mathrm{Re}$ & 75 & 0.223 & 0.7281 & 1.2678 & 0.424 \\
\hline $\mathrm{Os}$ & 76 & 0.226 & 0.7274 & 1.2675 & 0.431 \\
\hline $\mathrm{Ir}$ & 77 & 0.229 & 0.7266 & 1.2672 & 0.438 \\
\hline $\mathrm{Bi}$ & 83 & 0.249 & 0.7212 & 1.2650 & 0.466 \\
\hline & & & \\
\hline
\end{tabular}




\section{REFERENCES}

1. H.D. Zeman, E.B. Hughes, L. Campbell-Finman, R. Hofstadter, A. Hudson, J.N. Otis, J. Rolfe, E. Rubenstein, D.C. Harrison, R.S. Kernoff, A.C. Thompson, and G.S. Brown, Nucl. Inst. and Meth. 222 (1984), 308-318.

2. E. Rubenstein, R. Hofstadter, H.D. Zeman, A.C. Thompson, J.N. Otis, G.S. Brown, J.C. Giacomini, H.J. Gordon, R.S. Kernoff, D.C. Harrison, and W. Thomlinson, Proc. Natl. Acad. Sci. USA 83 (1986), 9724-9728.

3. W. Thomlinson, D. Chapman, N. Gmür, and N. Lazarz, Nucl. Inst. and Meth. in Phys. Res. A266 (1988), 226-233.

4. J.H. Hubbell, Nat. Stand. Ref. Data. Ser., Nat. Bur. Stand. NSRDS-NBS $29(1969), 64$.

5. H.D. Zeman, J.N. Otis, R. Hofstadter, A.C. Thompson, G.S. Brown, W. Thomlinson, E. Rubenstein, J.C. Giacomini, H.J. Gordon, and R.S. Kernoff, Synchrotron Radiation Applications to Digital Subtraction Angiography (SYRDA), E. Burattini and A. Rindi editors, Italy (1988), 75-82.

6. W. Thomlinson, N. Gmür, H.D. Zeman, J.N. Otis, R. Hofstadter, A.C. Thompson, G.S. Brown, E. Rubenstein, J.C. Giacomini, H.J. Gordon, and R.S. Kernoff, Synchrotron Radiation Applications to Digital Subtraction Angiography (SYRDA), E. Burattini and A. Rindi editors, Italy (1988), 173-179.

7. A.K. Freund, Nucl. Instr. and Meth. in Phys. Res. A266 (1988), 461- 466.

8. J.H. Beaumont, and M. Hart, J. Phys. E: Sci. Inst. 7 (1974), 823-829.

9. J.R. Schneider, O.D. Goncalves, A.J. Rollason, U. Bonse, J. Lauer, and W. Zulehner, Nucl. Inst. and Meth. in Phys. Res. B29 (1988), 661-674. 
10. J.R. Schneider, H. Nagasawa, L.E. Berman, J.B. Haitings, D.P. Siddons, and W. Zulehner, Nucl. Inst. and Meth. in Phys. Res. A276 (1989), 636-642.

11. J.J. Fitzgerald, G.L. Brownell, and F.J. Mahoney, Mathematical Theory of Radiation Dosimetry, Gordon and Breach, New York (1967) 108, 228-229.

12. E. Unger, and F. Gutierrez, Invest. Radiol. 21 (1986), 802-807.

13. M. Magerstädt, O.A. Gansow, M.W. Brechbiel, D. Colcher, L. Baltzer, R.H. Knop, M.E. Girton, and M. Naegele, Magnetic Resonance in Medicine 3 (1986), 808-812.

\section{FIGURE CAPTIONS}

1) The mass attenuation coefficients of iodine, gadolinium, hafnium, bismuth, bone and water plotted against the X-ray energy.

2) The optimal signal to noise ratio achievable with $10^{6}$ total $X$-ray photons per pixel and a patient thickness equivalent to $20 \mathrm{~cm}$ of water, when the artery being imaged lies behind the left ventricle which has 10 times the thickness of the artery.

3) The signal to noise ratio under the same conditions as in Fig. 2 plotted against the dimensionless measure of contrast agent concentration, $A$. Results are given for contrast agents containing bismuth, hafnium, gadolinium, and iodine. The results are shown at the optimal relative intensity of the two beams, $U$. The squares indicate the points on the four curves where the total mass of contrast agent is the same as the optimal mass of iodine.

4) The ratio of the $\mathrm{X}$-ray flux available from a Si mosaic crystal to that available from a perfect $\mathrm{Si}$ crystal plotted vs. X-ray energy for various values of the mosaic distribution half-width $\Gamma$. 
5) The signal to noise ratio achievable through $20 \mathrm{~cm}$ of water, a ventricle 10 times the artery thickness, and a constant contrast agent mass equal to the optimal mass for iodine. $10^{6}$ photons per pixel are used for the iodine case. The lower curve is for constant photon count, the upper curve is for constant total absorbed X-ray dose in the $20 \mathrm{~cm}$ of water.

6) The $\mathrm{X}$-ray photon count per pixel for the $\mathrm{X}-17$ superconducting wiggler magnet synchrotron radiation source at NSLS operated with a ring current of $250 \mathrm{~mA}$. A $4 \mathrm{msec}$ exposure time per $0.25 \times 0.25 \mathrm{~mm}$ pixel is assumed. For the lower curve a perfec $\_\mathrm{Si}\langle 1,1,1\rangle$ crystal is used for the monochromator. For the upper curve, a $S:\langle 1,1,1\rangle$ crystal is used with a mosaic distribution half-angle of 3 arc seconds.

7) The signal to noise ratio achievable through $20 \mathrm{~cm}$ of water, a ventricle 10 times the artery thickness, and a constant contrast agent mass equal to the optimal mass for iodine is shown for the output of the $\mathrm{X}-17$ superconducting wiggler magnet synchrotron radiation beam at NSLS with a stored $2.5 \mathrm{GeV}$ electron beam of $250 \mathrm{~mA}$ and a wiggler magnet field of $5 \mathrm{~T}$. For the lower curve a perfect $\operatorname{Si}\langle 1,1,1\rangle$ crystal is used for the monochromator. For the upper curve, a $\operatorname{Si}\langle 1,1,1\rangle$ crystal is used with a mosaic distribution half-angle of 3 arc seconds.

8) The skin dose per $4 \mathrm{msec}$ image frame for the $\mathrm{X}-17$ beam line for $250 \mathrm{~mA}$ of $\mathrm{X}$-ray ring current and $0.25 \mathrm{~mm}$ resolution vs. the $\mathrm{X}$-ray energy. The lower curve is for perfect Si monochromator crystals. The upper curve is for mosaic crystals with a mosaic distribution half-width of 3 arc seconds. 
The Mass Attenuation Coefficient of Water, Bone, Iodine, Gadolinium, Hafnium, and Bismuth vs. X-Ray Energy

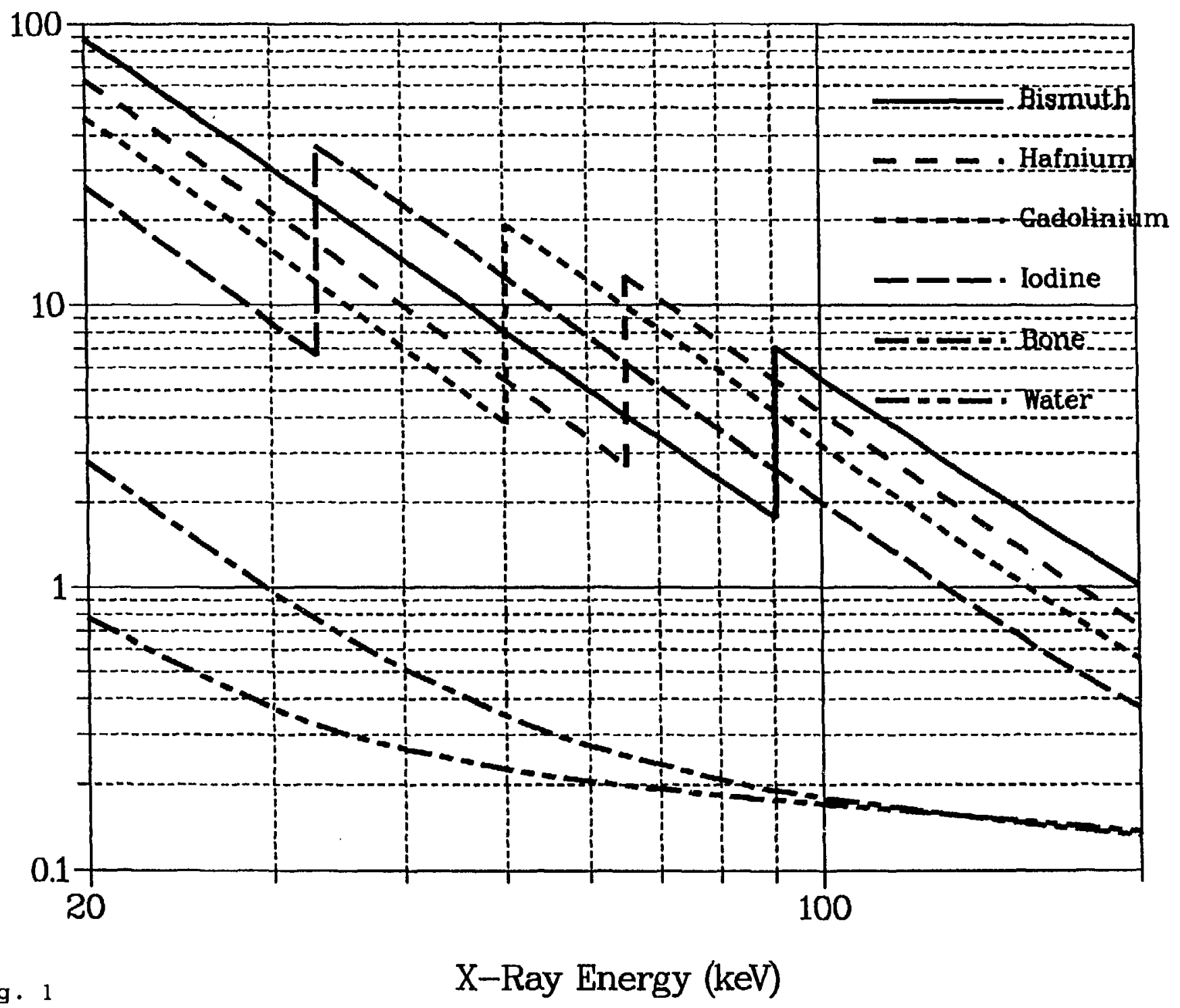


The Optimal Signal to Noise Ratio Looking Through $20 \mathrm{~cm}$ of Water and a Filled Ventricle 10 Times the Artery Thickness for $10^{6}$ Total Photons/Pixel

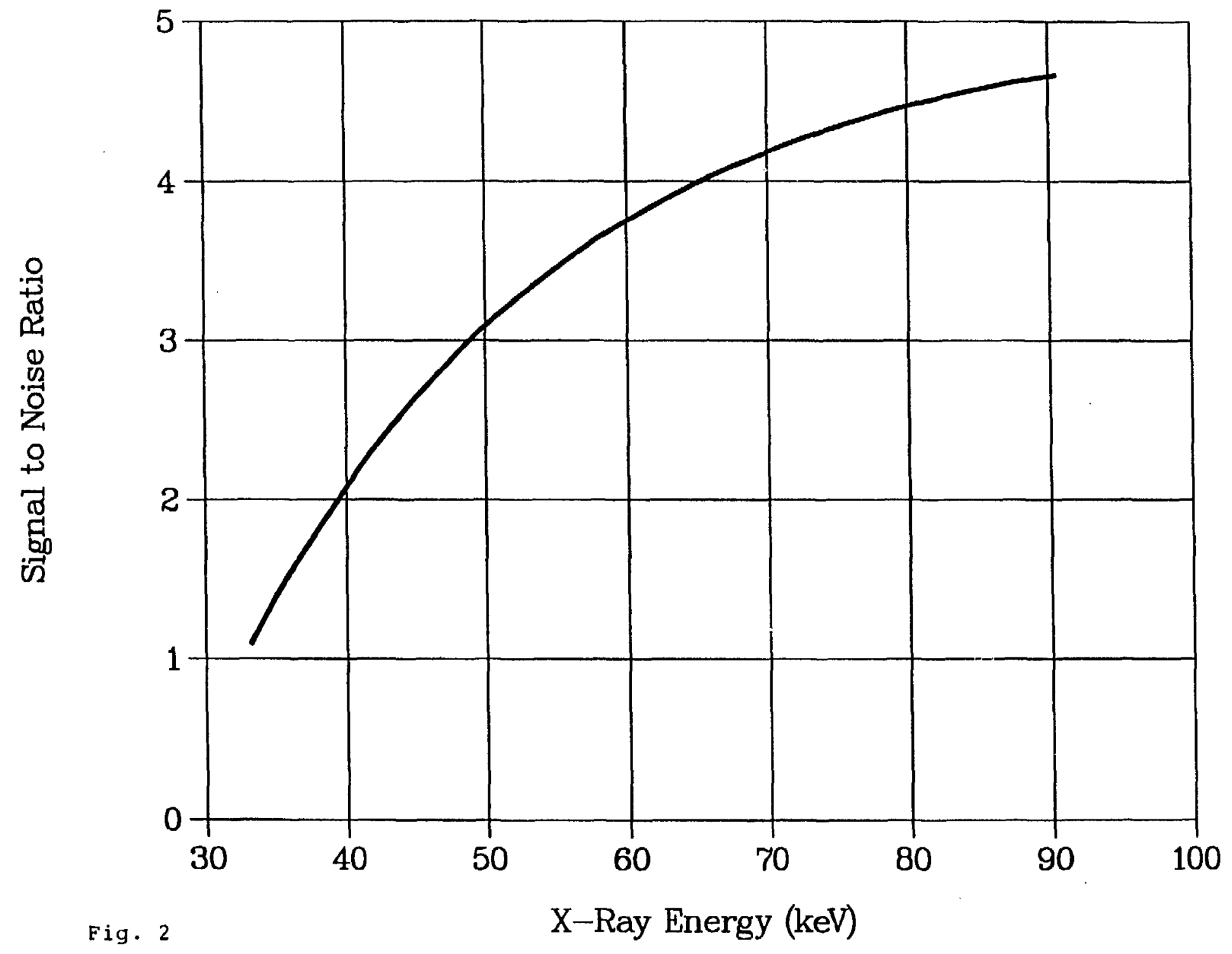


The Signal to Noise Ratio Looking Through $20 \mathrm{~cm}$ of Water and a Filled Ventricle 10 Times the Artery Thickness for $10^{6}$ Total Photons/Pixel

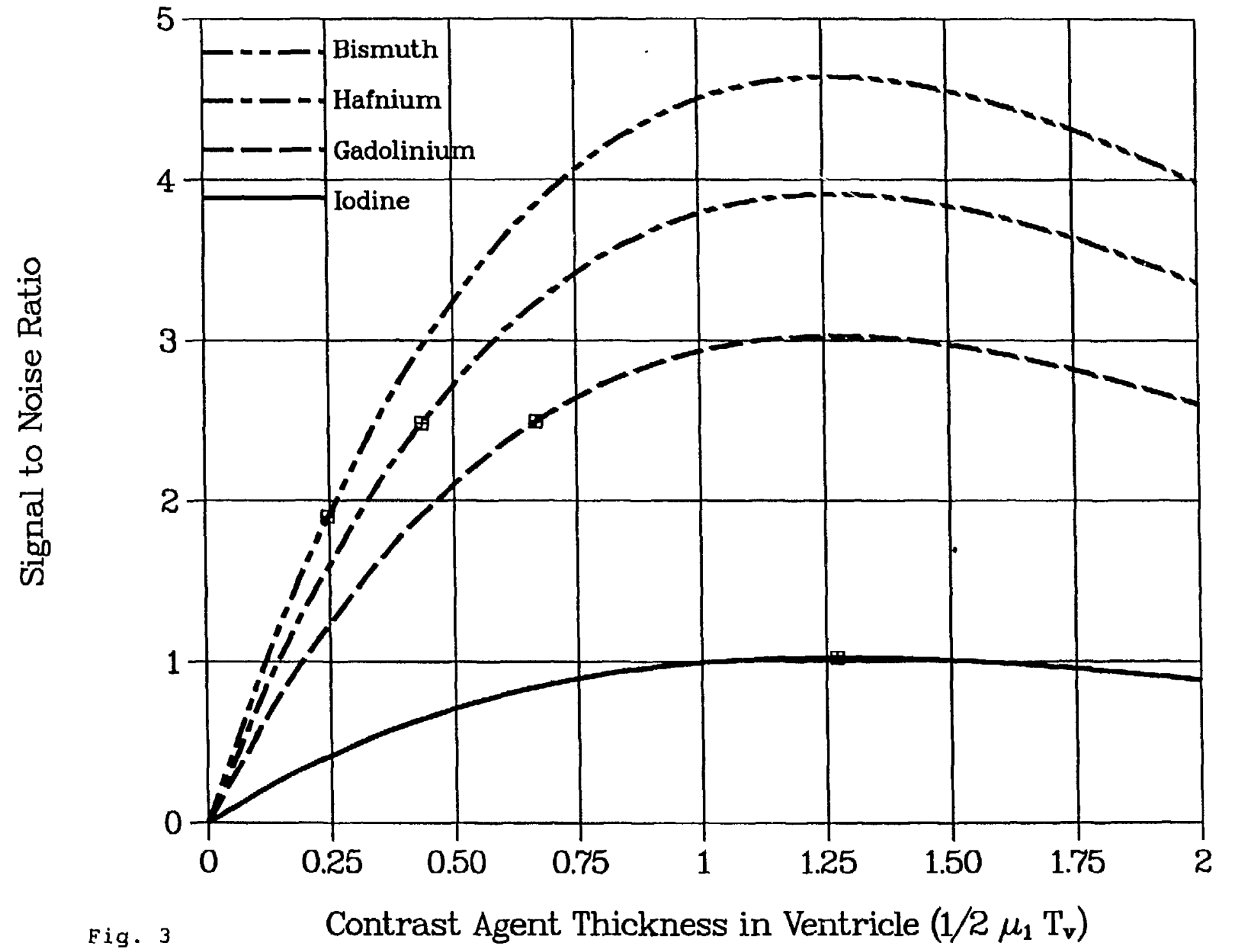


X-Ray Flux Enhancement Ratio for Si $(1,1,1)$ Mosaic Crystals Compared with Perfect Si $\{1,1,1\rangle$ Crystals vs X-Ray Energy

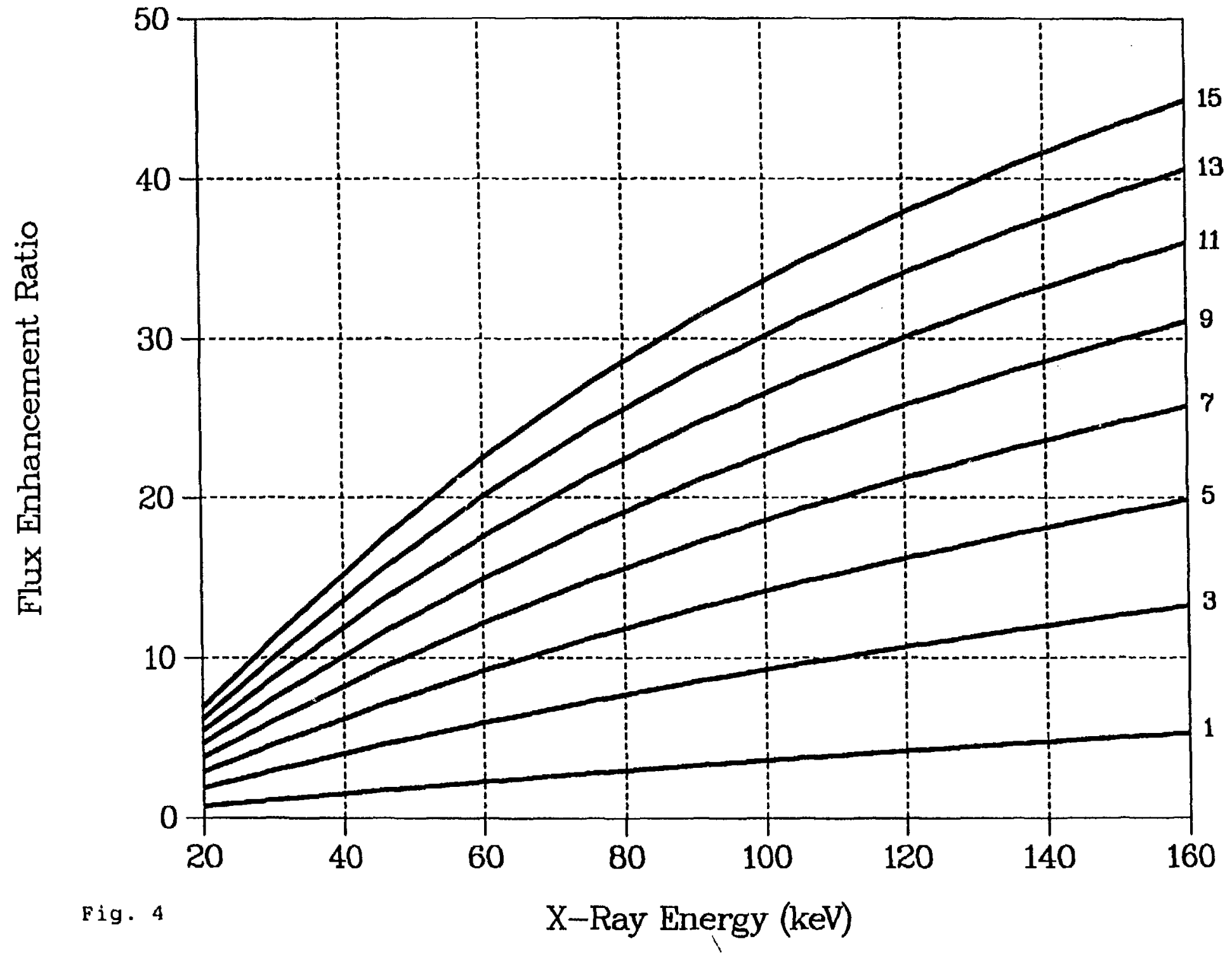


SNR with $20 \mathrm{~cm}$ of Water, a Ventricle 10 Times the Artery, $10^{6}$ Photons/Pixel (for I), and Constant Contrast Agent mg (Optimal for I)

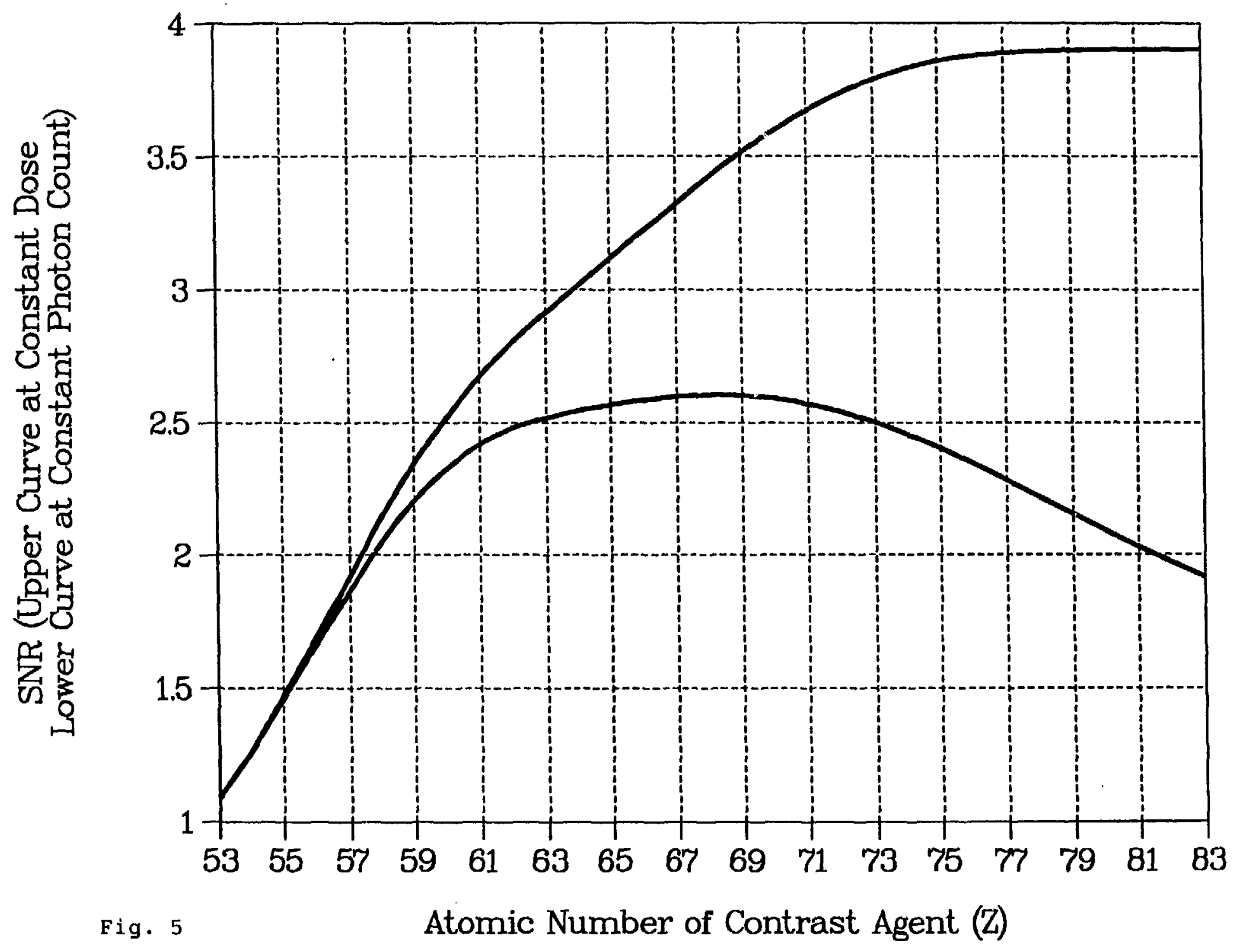


The X-Ray Photon Count per $0.25 \times 0.25 \mathrm{~mm}$ Pixel in $4 \mathrm{msec}$ for the X-17 Beam Line at $250 \mathrm{~mA}$ X-Ray Ring Current

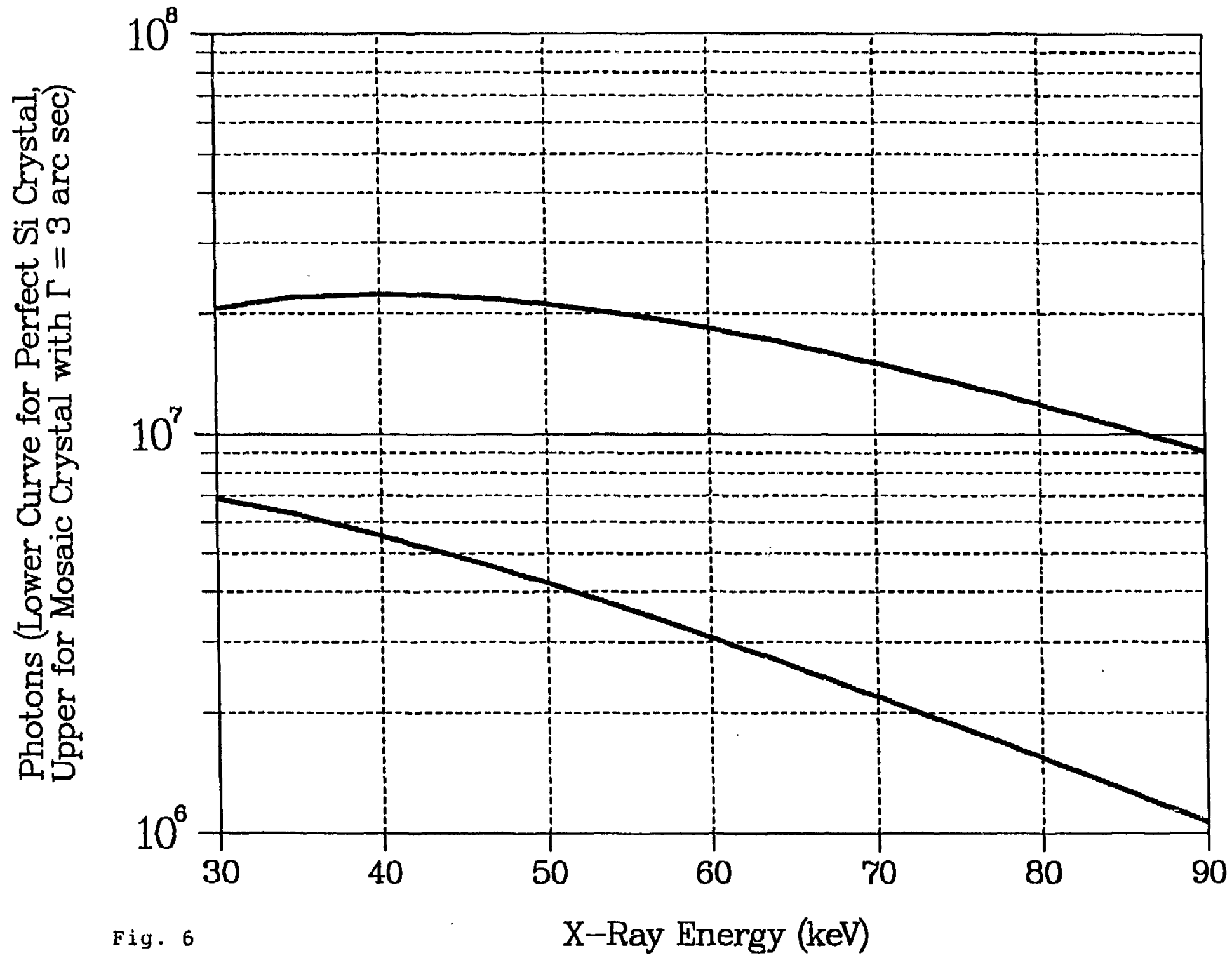


SNR at $X-17$ Beam Line with $20 \mathrm{~cm}$ of Water, a Ventricle 10 Times the Artery, $250 \mathrm{~mA}$ in X-Ray Ring, and Constant Contrast Agent $\mathrm{mg}$

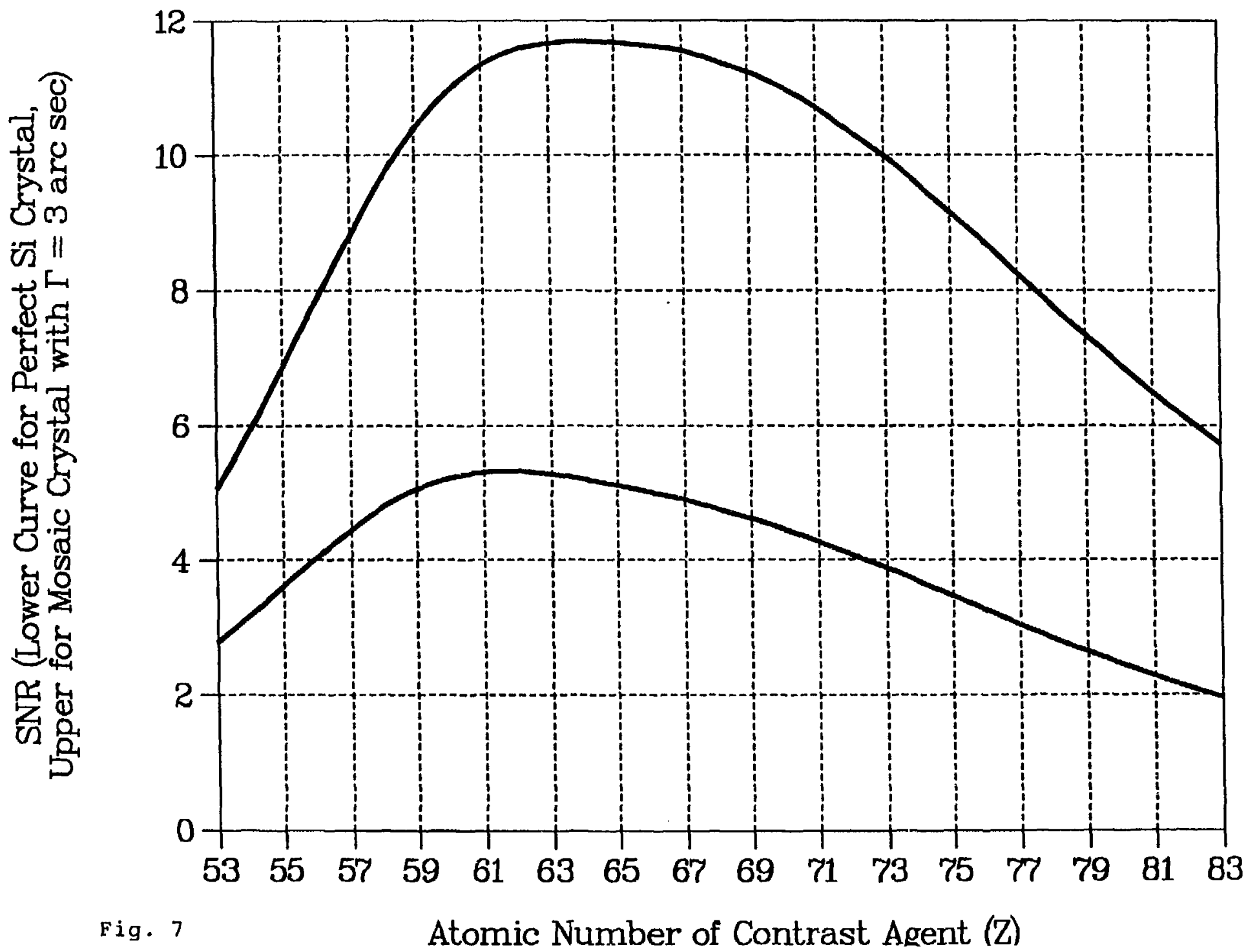


The Patient Skin Dose per $4 \mathrm{msec}$ Image Frame for the X-17 Beam Line at $250 \mathrm{~mA} \mathrm{X}$-Ray Ring Current

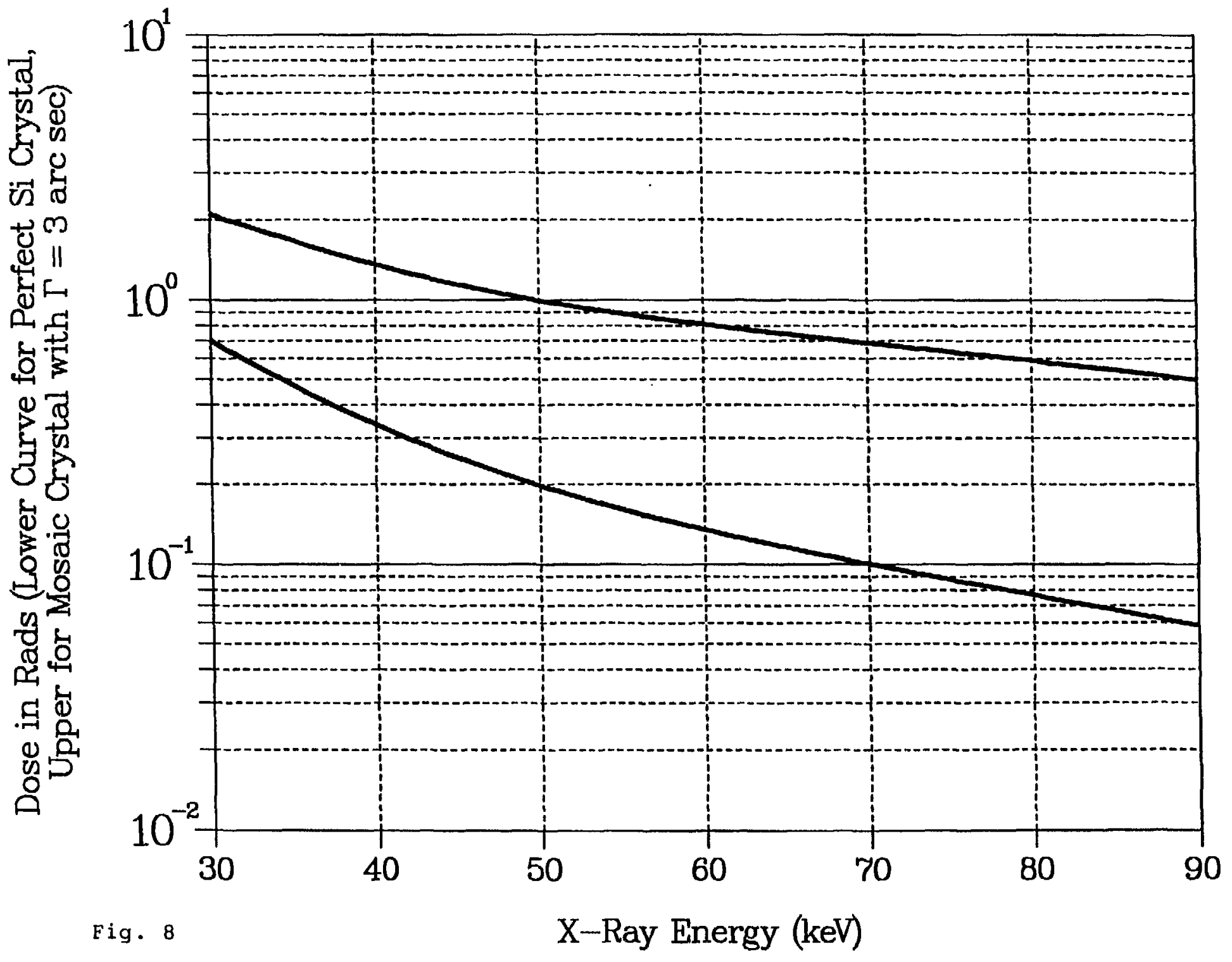

